\title{
Live twin birth after successful treatment of a ruptured heterotopic pregnancy by laparoscopy
}

\author{
Murat Gözüküçük • Şerife Esra Çetinkaya • \\ Batu Aydınuraz • Korhan Kahraman • \\ Mustafa Hakan Șatıroğlu
}

Received: 12 December 2008 / Accepted: 23 February 2009 / Published online: 10 March 2009

(C) Springer-Verlag 2009

\begin{abstract}
We report a case of a live twin birth after laparoscopic treatment of a ruptured heterotopic pregnancy. A 29-year-old woman, with a history of right salpingectomy for ectopic pregnancy, became pregnant after transfer of three embryos at in vitro fertilization treatment. At the ninth week of gestation, she was admitted to our clinic with abdominal pain. Ultrasonographic examination revealed a triplet heterotopic pregnancy consisting of an intrauterine twin pregnancy and an ectopic pregnancy in the left fallopian tube. An immediate laparoscopy was planned and left salpingectomy was performed. In the postoperative period, intrauterine twin pregnancy continued uneventfully; at the 35th week of gestation, two healthy infants with birth weights of 2,206 and 2,426 g were delivered. Heterotopic pregnancies must be kept in mind after assisted reproductive techniques. Early diagnosis allows successful laparoscopic treatment without sequel. Laparoscopic surgery is an appropriate method to manage selected patients with heterotopic tubal pregnancy.
\end{abstract}

Keywords Heterotopic pregnancy.

Ruptured tubal pregnancy - Laparoscopy· Live twin birth

M. Gözüküçük $(\bowtie) \cdot$ Ş. E. Çetinkaya $\cdot$ K. Kahraman •

M. H. Șatıroğlu

Department of Obstetrics and Gynecology, Medical Faculty,

Ankara University,

Cebeci,

Ankara, Turkey

e-mail: muratgozukucuk@gmail.com

B. Aydinuraz

Women's Health and Reproductive Medicine Center, HS Clinic,

Ankara, Turkey

\section{Introduction}

Ectopic pregnancy (EP) refers to the implantation of a viable ovum outside the uterine corpus. Heterotopic pregnancy (HP) is the simultaneous occurrence of gestations at two or more implantation sites. It is most often manifested as concomitant intrauterine pregnancy (IUP) and EP [1]. Although EP is not uncommon in women of reproductive age, HP is rare in the general population, with an incidence of 1:7,963-30,000 in spontaneous conceptions [2]. The increased incidence of pelvic inflammatory disease (PID), the common usage of ovulation inducing agents, and assisted reproductive techniques (ART) have contributed to the increasing incidence of both multiple gestations and HP in the last decade. The rate of HP after in vitro fertilization (IVF) has been reported to be as high as $1 \%[2,3]$, although it's true incidence is unknown. Most recent studies exhibit the incidence about 152 per 100,000 pregnancies in ART cycles [4]. Due to the difficulty in the diagnosis, rupture of the tube, bleeding, and the need for emergency operations are seen more often in heterotopic than in ectopic pregnancies. Thus, all pregnancies as a result of ART must be evaluated cautiously. Here, we report a triplet heterotopic pregnancy, which was successfully diagnosed and treated by laparoscopy.

\section{Case}

A 29-year-old woman G: 1, P: 0, with unexplained infertility, not to be able to conceive for 3 years, underwent an IVF procedure. She had a history of an EP 3 years ago treated by unilateral right salpingectomy. After initial downregulation using leuprolide acetate $500 \mu \mathrm{g}$ /day (Lucrin; Abbott, Cedex, Istanbul, Turkey), 225 IU/day of recombinant follicle stimulating hormone (Gonal F; Serono 
Laboratories) was given starting from the second day of the menstrual cycle. When at least three follicles $>17 \mathrm{~mm}$ were observed, human chorionic gonadotropin (hCG; Pregnyl; Organon, Cambridge, UK) was administered 10,000 IU i.m. and oocyte retrieval was performed at $35.5 \mathrm{~h}$. Five of eight retrieved oocytes were successfully fertilized by IVF. Two days after oocyte retrieval, three embryos were transferred. Luteal phase support in the form of intravaginal micronized progesterone was given. Serum beta-hCG was $52 \mathrm{IU} / \mathrm{mL}$ on day 12 and we deduced intrauterine live twin pregnancy 4 weeks after embryo transfer.

At the ninth week of gestation, she was admitted with abdominal pain. Transabdominal ultrasonography (US) examination revealed intrauterine live twin pregnancy and a left-sided ectopic pregnancy with fetal heart beat. The vital signs of the patient were in normal range. A diagnostic laparoscopy was performed using general anesthesia and the ports were placed in classical locations carefully to protect the uterus. A ruptured left tubal EP was found covered with omentum hanging on the anterior abdominal wall (Figs. 1 and 2). Left salpingectomy was performed successfully. The patient was discharged at the second day after the operation. In the postoperative period, the intrauterine twin pregnancy continued uneventfully with no unusual aspect of the prenatal care and she delivered two healthy infants at the 35th week of gestation with birth weights of 2,206 and 2,426 g.

\section{Discussion}

HP is a rare entity in spontaneous cycles; however, its incidence has risen with the widespread use of ART. Although most ectopic gestations in HPs occurring after ART are tubal, $10.8 \%$ are extratubal [5] and more difficult

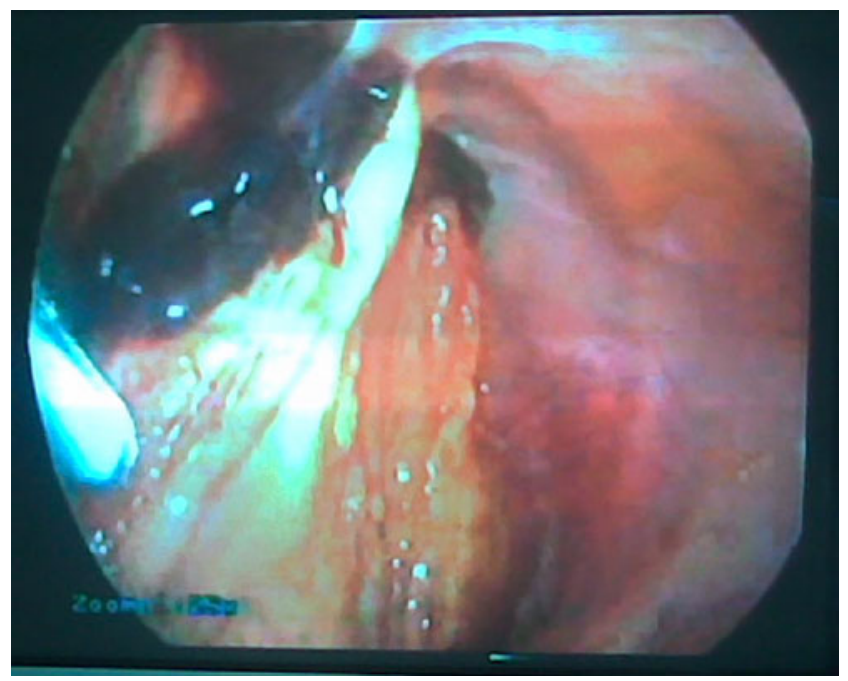

Fig. 1 Ruptured gestational sac localized at the left tube surrounded with omentum

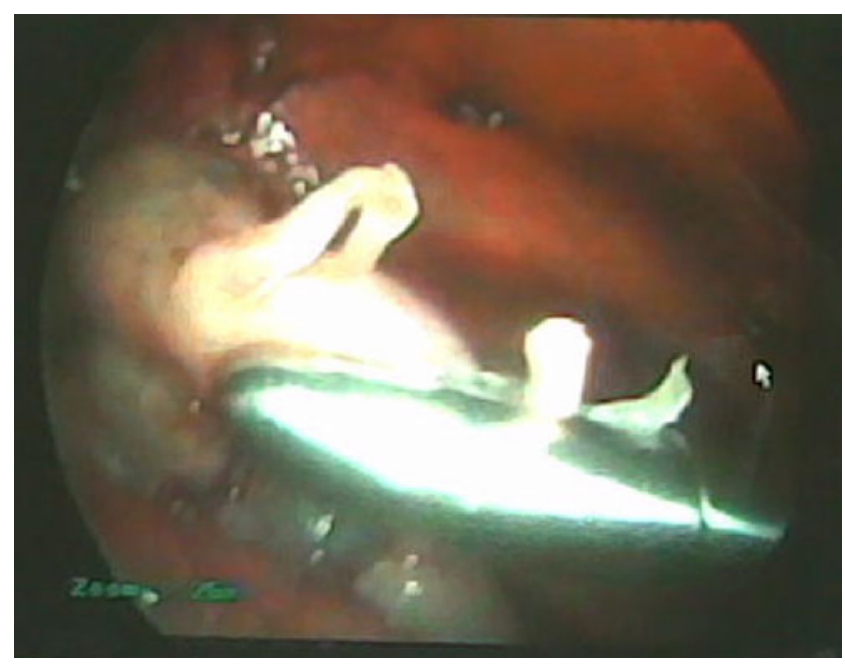

Fig. 2 Floating fetus in abdominal fluid after detachment of the omentum

to diagnose. The beta-hCG may continue to rise normally and US may be unreliable in the presence of a normal intrauterine gestation especially in HPs. The intermittent unilateral pain can be attributed to a hemorrhagic corpus luteum, or a small degree of ovarian hyper stimulation [6]. Therefore, we need to have better evaluation of the adnexa when pain is out of proportion of what is expected in a woman with IUP. Most cases are missed on their initial examination, and patients frequently present with symptoms of rupture before the diagnosis is ultimately made, as in this case. Therefore, keeping its high incidence after ART in mind, careful surveillance of extrauterine structures at the first US examination is essential in pregnancies achieved after ART.

Different mechanisms may predispose to development of HP after ART cycles. More embryos transferred by ART procedures increase both multiple gestation and EP incidences [7]. Other factors predisposing to ectopic gestation are previous tubal damage caused by PID, endometriosis and tubal surgery, previous ectopic pregnancy, and ovulation induction. The hormonal milieu at the moment of transfer has been proposed as possible causes as well [8]. Thus, it seems more important to limit the number of embryos transferred, particularly in patients who present risk factors for HP and in young women, thereby minimizing the risk of EP.

Concerning the prognosis of the IUP, favorable outcomes are reported in 50-60\% of cases [2]. In HPs, spontaneous or induced abortions are more likely to occur than with intrauterine-only pregnancies; however, birth outcomes are similar, when an intrauterine gestation of a heterotopic pregnancy results in a live birth [9].

Management of HP should be conservative if possible; the primary goal is removal of the $\mathrm{EP}$, while preserving the intrauterine pregnancy. Several treatment modalities have been described, either surgical or medical. The choice of treatment depends mainly on the hemodynamic condition 
of the patient, localization of the ectopic pregnancy, and desire for the ongoing pregnancy and future pregnancies.

The safety of laparoscopy during pregnancy has been well documented [1]. The advantages of laparoscopy compared with laparotomy in postoperative recovery are well known [1]. Laparoscopy allows prompt diagnosis and treatment, thereby providing good outcome avoiding the postoperative inconvenience of laparotomy, and has the advantage of an immediate result compared with medical treatment. In our case, the pregnancy was not affected by the operation and resulted with delivery of healthy twins.

Moreover, every physician dealing with ART should be aware of the possibility of HP, even in the absence of any predisposing risk factors. A high index of suspicion followed by an early surgical laparoscopic intervention can minimize maternal morbidity and preserve continuing IUP.

\section{References}

1. Louis-Sylvestre C, Morice P, Chapron C, Dubuisson JB (1997) The role of laparoscopy in the diagnosis and management of heterotopic pregnancies. Hum Reprod 12:1100-1102
2. Barrenetxea G, Barinaga-Rementeria L, Lopez de Larruzea A et al (2007) Heterotopic pregnancy: two cases and a comparative review. Fertil Steril 87:e9-e15

3. Chin HY, Chen FP, Wang CJ et al (2004) Heterotopic pregnancy after in-vitro fertilization-embryo transfer. Int $\mathrm{J}$ Gynecol Obstet $86: 411-416$

4. Clayton HB, Schieve LA, Peterson HB, Jamieson DJ, Reynolds MA, Wright VC (2006) Risk of ectopic pregnancy among women who underwent ART, United States, 1999-2001. Obstet Gynecol 107:595-604

5. Tal J, Haddad S, Gordon N, Timor-Tritsch I (1996) Heterotopic pregnancy after ovulation induction and assisted reproductive technologies: a literature review from 1971 to 1993 . Fertil Steril $66: 1-12$

6. Nikolaou DS, Lavery S, Bevan R et al (2002) Triplet heterotopic pregnancy with an intrauterine monochorionic diamniotic twin pregnancy and an interstitial pregnancy following in-vitro fertilisation and transfer of two embryos. J Obstet Gynaecol 22:94-95

7. Pan HS, Chuang J, Chiu SF et al (2002) Heterotopic triplet pregnancy: report of a case with bilateral tubal pregnancy and an intrauterine pregnancy. Hum Reprod 17:1363-1366

8. Paltieli Y, Eibschitz I, Ziskind G et al (2000) High progesterone levels and ciliary dysfunction - a possible cause of ectopic pregnancy. J Assist Reprod Genet 17:103-106

9. Clayton HB, Schieve LA, Peterson HB et al (2007) A comparison of heterotopic and intrauterine-only pregnancy outcomes after assisted reproductive technologies in the United States from 1999 to 2002. Fertil Steril 87:303-309 